\title{
FLUKA Simulations for SEE Studies of Critical LHC Underground Areas
}

\author{
Ketil Røed, Vittorio Boccone, Markus Brugger, Alfredo Ferrari, Daniel Kramer, Elias Lebbos, Roberto Losito,
} Alessio Mereghetti, Giovanni Spiezia, and Roberto Versaci

\begin{abstract}
FLUKA Monte Carlo simulations have been performed to identify particle energy spectra and fluences relevant for evaluating the risk of single event effects in electronics installed in critical LHC underground areas. Since these simulations are associated with significant uncertainties, the results will compared with an online monitoring system installed to evaluate radiation levels at the location of the electronics. This comparison approach have been benchmarked in a mixed field reference facility and for a preliminary LHC monitoring case study.
\end{abstract}

Index Terms-CERN, FLUKA, LHC, Monte Carlo, radiation effects, SEU.

\section{INTRODUCTION}

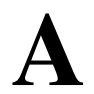
T the Large Hadron Collider (LHC) located at CERN radiation levels in various critical areas have been analyzed by Monte Carlo simulations. These LHC alcoves are partly equipped with commercial electronic devices not specifically designed to be radiation tolerant. It is therefore important to determine the respective particle energy spectra and fluences in these areas to identify the associated risk of failure due to radiation and possible mitigation options, and to define appropriate test criteria for the equipment. Depending on the location, the main radiation effects of concern are related to the steady accumulation of defects such as total integrated dose or displacement damage, and Single Event Effects (SEEs), e.g., Single Event Upsets (SEUs). In the present configuration, the commercial electronics in the LHC alcoves are mostly affected by the risk of SEUs, while electronics installed in the LHC tunnel will additionally suffer from cumulative effects. However, as measures already have been taken to improve the radiation tolerance of tunnel electronics, this paper will focus on the particle energy spectra and fluences relevant for SEU studies of electronics in the LHC alcoves. For radiation environment studies concerning detectors and electronics for CERN experiments the reader is referred to for example [1]-[5].

The LHC is already a record breaking machine being the only accelerator to have accelerated and collided protons at a kinetic energy of $3.5 \mathrm{TeV}$. Pursuing a target beam energy of

Manuscript received September 17, 2010; revised November 15, 2010; accepted November 22, 2010. Date of publication February 17, 2011; date of current version June 15, 2011.

The authors are with CERN, CH-1211 Genève 23, Switzerland (e-mail: ketil. roeed@cern.ch).

Color versions of one or more of the figures in this paper are available online at http://ieeexplore.ieee.org.

Digital Object Identifier 10.1109/TNS.2010.2097605
$7 \mathrm{TeV}$ and luminosity of $10^{34} \mathrm{~cm}^{-2} \mathrm{~s}^{-1}$ [6], makes the operation and layout of LHC is extremely complex. Monte Carlo simulations to determine radiation levels in LHC underground areas are therefore associated with significant uncertainties. A multi-node monitoring system is therefore installed around the LHC to provide an online evaluation of the radiation levels at the location of the electronics. The possibility and quality of comparing the simulations with the measurements is demonstrated in Section III by a well controlled application benchmark, and in Section V by a preliminary LHC monitoring case study.

Due to the various shielding configurations (e.g., concrete and/or iron walls) separating the LHC alcoves from the beam line and tunnel, a significant thermal neutron fluence is expected. For the majority of devices installed in the alcoves, the sensitivity to thermal neutrons is unknown. In [7] it is shown how the thermal neutron SEU cross sections can vary orders of magnitude between different types of devices. A so-called 'risk-factor', $R_{\mathrm{Th}}$, giving the ratio of the thermal neutron fluence to the high-energy hadron fluence, has therefore been introduced in order to identify thermal neutron critical areas.

\section{The FLUKA Monte CARlo CoDE}

The simulations presented in this paper have been performed using the FLUKA Monte Carlo code (version 2008.3d.1). FLUKA is a well benchmarked [8]-[11] general purpose tool for calculations of particle transport and interactions with matter, covering an extended range of applications like for example proton and electron accelerator shielding, target design, calorimetry, activation and dosimetry, cosmic ray studies, and radiotherapy. It can simulate with high accuracy the interaction and propagation in matter of about 60 different particles, including photons and electrons from $1 \mathrm{keV}$ to thousands of $\mathrm{TeV}$, neutrinos, muons of any energy, and hadrons of energies up to $20 \mathrm{TeV}$. At CERN FLUKA is extensively used for machine protection issues such as energy deposition calculations and studies of material damage to accelerator elements [12]. A very detailed library of material and geometry descriptions is consequently available for accelerator elements like for example magnets and collimators. Based on this library beam lines and tunnel regions are built to study particle fluences and energy spectra relevant for SEUs in the electronics installed in various LHC alcoves.

Due to the complexity of the beam line geometry the calculation time needed to reach sufficient statistics is often a bottleneck. As the focus of this paper is on SEUs and not total ionizing dose, the simulations presented have been optimized in terms of calculation time by disabeling the transport of electrons and photons, effectively turning off the electromagnetic 
cascade. The transport cut-off for charged hadrons has been set to $20 \mathrm{MeV}$. This is justified by that rapid decrease in the charged hadron nuclear interaction cross section from about $20 \mathrm{MeV}$ and below. Also, as the energy decreases the charged hadrons will no longer be able to penetrate the device package to reach the sensitive area. Below $20 \mathrm{MeV}$ the contribution from charged hadrons is therefore considered to be insignificant. This threshold, however, does not apply to neutrons which may contribute down to the nuclear interaction threshold of the respective materials. Neutrons from $20 \mathrm{MeV}$ and down to some few $\mathrm{MeV}$ should therefore be included when calculating the expected SEU rate. Also, considering that the LHC electronics may have been designed and developed many years ago, it is likely that some devices may contain boron as a p-type dopant or from the formation of boron-doped phosphosilicate glass (BPSG) dielectric. Boron is composed of two isotopes, ${ }^{11} \mathrm{~B}(80.1 \%$ abundance $)$ and ${ }^{10} \mathrm{~B}$ (19.9\% abundance). The ${ }^{10} \mathrm{~B}$ is unstable when exposed to neutrons and breaks into a ${ }^{7} \mathrm{Li}$-recoil and $\alpha$-particle with sufficient ionization power to induced SEUs [13]. At thermal energies the capture cross section is approximately 4000 barns, which is four orders of magnitude higher than the $\alpha$-particle production cross section from high energy neutrons in silicon. Neutrons are therefore transported down to thermal energies in order to evaluate the expected fluences and associated risk of thermal neutrons.

For the purpose of the simulation studies presented in this paper, hadrons above $20 \mathrm{MeV}$ (high energy hadrons) are considered to be equally effective in inducing SEUs due to their approximately similar nuclear interaction cross section. Even though some differences exist between the respective cross sections, these differences are limited to the $\Delta$-resonance region for pions, and to lower energies where the coulomb repulsion starts to affect the charged hadrons [14]. In addition to protons, neutrons and pions, kaons are also considered to be part of the high energy hadron group. However, as the production yield of kaons is in the order of a few percent they are not considered to be a major contributor to the SEU rate. Moreover, the radiation environment in the LHC alcoves is typically dominated by neutrons.

\section{APPLICATION BENCHMARK}

To demonstrate the method of using FLUKA to predict response of a dedicated SEU detector in a mixed radiation field, a benchmark experiment was carried out at the well controlled CERN-EU high Energy Reference Field facility (CERF) [15]. At this facility a $120 \mathrm{GeV} / \mathrm{c}$ mixed hadron beam $\left(60.7 \% \pi^{+}, 34.8 \% \mathrm{p}, 4.5 \% \mathrm{~K}^{+}\right)$, from the Super Proton Synchrotron (SPS) accelerator, is aimed at a $50 \mathrm{~cm}$ Copper target creating a particle energy spectra similar to what can be expected in some of the underground areas in the LHC. FLUKA simulations have been performed to determine the fluence of High Energy Hadrons (HEH), 5-20 MeV neutrons, and thermal neutrons in 8 different positions upstream, next to, and downstream of the Copper target. Fig. 1 shows how the most downstream positions 4,5 , and 6 are dominated by the high energy hadrons, and the upstream positions 2, 1, C150, and $\mathrm{C} 250$ are dominated by thermal neutrons. Position 3 is

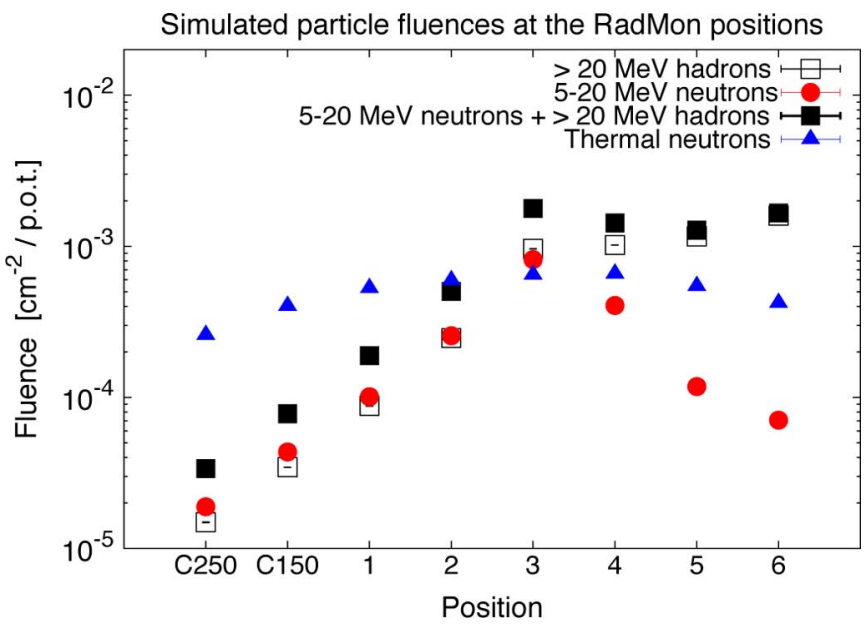

Fig. 1. Particle fluences determined by FLUKA simulations at the various RadMon measurement positions. The values are normalized to the number of particles on target (p.o.t.). The statistical errors of the simulations are less than $1-2 \%$.

TABLE I

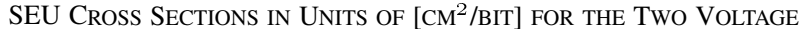
SETTINGS USED FOR THE RADMON INSTALLED IN THE LHC [18], [19]. THE ASSOCIATED UNCERTAINTIES OF 10\% ARE DOMINATED BY BEAM MONITORING

\begin{tabular}{l|c|c|c}
\hline \hline Bias & $\sigma_{H E H}\left[\mathrm{~cm}^{2} / \mathrm{bit}\right]$ & $\sigma_{T h}\left[\mathrm{~cm}^{2} / \mathrm{bit}\right]$ & $\frac{\sigma_{T h}}{\sigma_{H E H}}$ \\
\hline $3 \mathrm{~V}$ & $7.0 \cdot 10^{-14} \pm 10 \%$ & $1.7 \cdot 10^{-13} \pm 10 \%$ & 2.4 \\
$5 \mathrm{~V}$ & $3.0 \cdot 10^{-14} \pm 10 \%$ & $3.1 \cdot 10^{-15} \pm 10 \%$ & 0.1 \\
\hline \hline
\end{tabular}

located next to the target with a high contribution from all the three fluences of interest. The response of a dedicted SEU radiation monitor (RadMon [16]) is then compared with FLUKA simulations for the respective locations. This monitor is based on 4 Toshiba TC554001AF-70L 4 Mbit SRAM memory devices. Using SRAM devices as an SEU monitor has also been demonstraded by the ESA Reference SEU Monitor presented in [17]. In addition the RadMon comprises a RadFET and PIN diode relevant to measurements of total dose and displacement damage which is not the subject of this paper. Relevant SEU cross sections for the Toshiba SRAM memory device are listed in Table I. The high energy hadron cross sections are based on previous measurements using monoenergetic proton beams and quasi-monoenergetic neutron beams, while the thermal neutron cross sections are based on epithermal neutron beam measurements [18]. For the latter, a cadmium filter was used to discriminate between the pure low energy neutron component $(<0.5 \mathrm{eV})$ and the total neutron fluence [18]. The bit cross sections were typically calculated by measuring the total number of SEUs over all 4 memory devices and then normalizing the result to the total number of bits and beam fluence.

The RadMon can be operated at two different voltage settings in order to achieve different sensitivities to both thermal neutrons and high-energy hadrons. In total several hundreds of RadMons are placed in strategic locations around the LHC tunnel to monitor the radiation field relevant to radiation induced failures in LHC electronics. In addition they provide valuable information for the benchmarking of FLUKA.

For the application benchmark experiment at CERF, the SEU response of 2 identical RadMons were predicted for $3 \mathrm{~V}$ and 5 


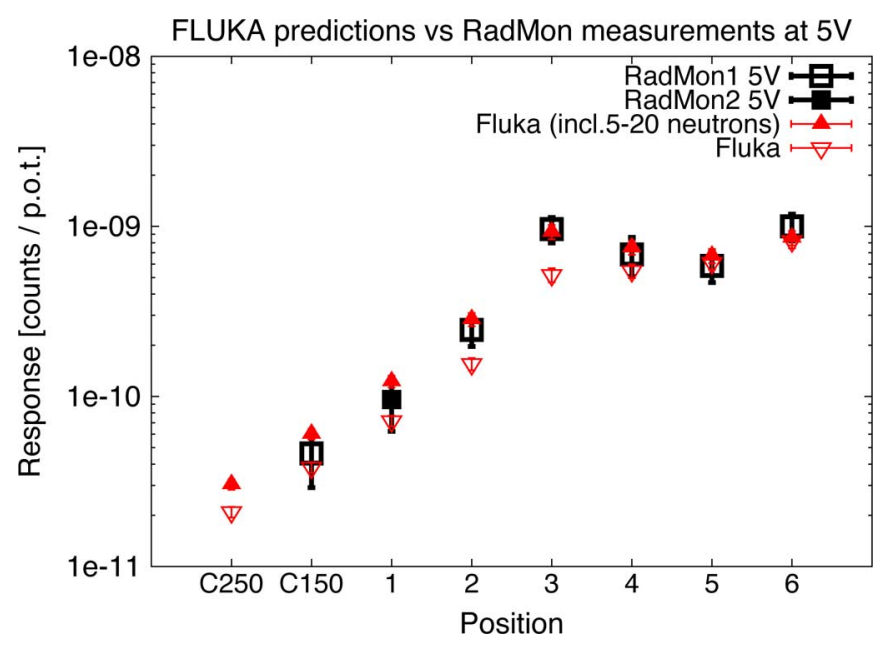

Fig. 2. FLUKA response predictions compared to the measured response of 2 RadMons at $5 \mathrm{~V}$. All results are normalized to the number of particles on target. For the predictions the error bars reflect statistical uncertainties of the simulation in addition to the uncertainties associated with the cross section values used. For the RadMon measurements the error bars reflect statistical uncertainties only.

$\mathrm{V}$ operation by combining FLUKA simulation results with the measured SEU cross sections of the RadMons as shown in (1).

$$
\text { Response }=\Phi_{\mathrm{HEH}} \cdot \sigma_{\mathrm{HEH}}+\Phi_{\mathrm{Th}} \cdot \sigma_{\mathrm{Th}} \cdot
$$

With the previous definition of high energy hadrons, this assumes no sensitivity to hadrons below $20 \mathrm{MeV}$. As already discussed in Section II, this is not true for neutrons as they are not affected by the coulomb barrier. In fact, recent measurements have demonstrated that the RadMon has a significant SEU sensitivity when irradiated with neutrons down to $5.8 \mathrm{MeV}$. The analysis of these measurements is ongoing and will be published at a later stage. For the moment the sensitivity to $5-20 \mathrm{MeV}$ neutrons is assumed to be equal to and grouped with high energy hadrons when included for the comparisons presented in Figs. 2 and 3. When a RadMon is operated at $5 \mathrm{~V}$ its measured response closely follows the trend of the high energy hadron fluence and shows no significant dependence on the thermal neutron fluence. For position 3 it can further been seen how the RadMon also is sensitive to neutrons in the energy range $5-20 \mathrm{MeV}$. Changing the biasing from $5 \mathrm{~V}$ to $3 \mathrm{~V}$ operation, the relative sensitivity to thermal neutrons is increased by a factor 24 compared to the high energy hadron fluence. Thus, at $3 \mathrm{~V}$ the response of the RadMon is dominated by thermal neutrons. This effect is evident for the most upstream positions where the response curve no longer have the same rapid decrease as for the $5 \mathrm{~V}$ case. By changing the voltage the RadMon can therefore be used to discriminate between the thermal neutron fluence and high energy hadron fluence as described in [18].

Overall the agreement between the predicted and measured response is within $10-30 \%$ for RadMon 1 while RadMon 2 seems to have systematic lower response for all measured positions. This offset is currently believed to be due to variations in the sensitivity between the individual SRAM memories used in the RadMons. Dedicated mono-energetic irradiation tests have therefore been scheduled in the near future to investigate and

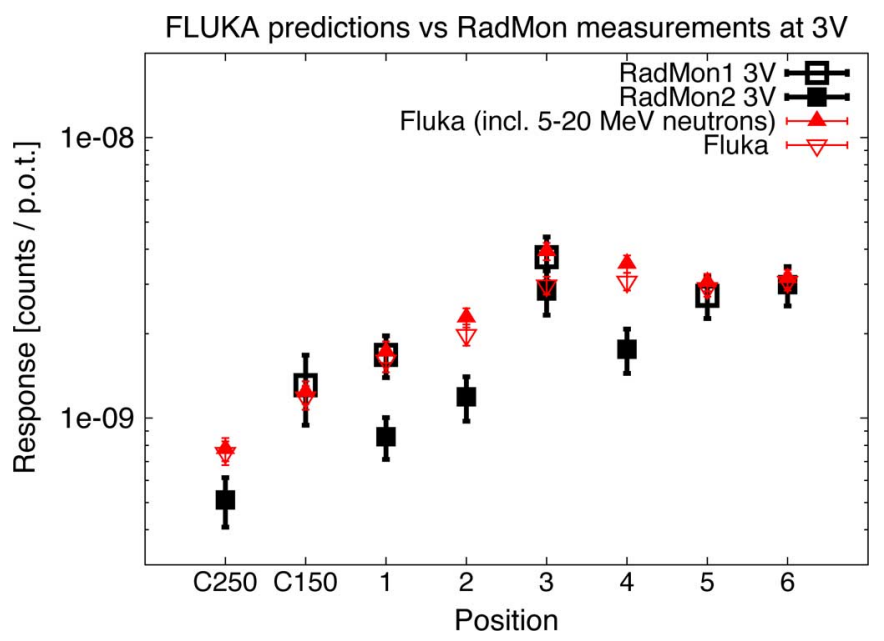

Fig. 3. FLUKA response predictions compared to measured response or 2 RadMons at $3 \mathrm{~V}$. All results are normalized to particles on target. For the predictions the error bars reflect statistical uncertainties of the simulation in addition to the uncertainties associated with the cross section values used. For the RadMon measurements the error bars reflect statistical uncertainties only.

possible quantify this variation by single component irradiations. The outcome of these tests will then be used to decrease the uncertainty at which the RadMons can measure the radiation level at the LHC. Nevertheless, all measurements at CERF are within a factor 2 of the response predicted by FLUKA simulations combined with previously measured SEU cross sections. This is considered acceptable for the purpose of this study and for the practical use of the RadMon.

\section{OVERVIEW of LHC CRITICAL AREAS}

\section{A. Sources of Radiation and Uncertainties}

The main sources of radiation relevant to radiation effects in electronics at the LHC are direct losses in collimator and collimator like objects, particle debris from proton-proton or lead-lead collisions in the four main experiments, and interaction of the beam with the residual gas inside the beam pipe. The radiation levels are expected to scale with the beam intensity for direct losses, with luminosity for collision debris, and with both beam intensity and residual gas density for beam-gas interactions. While direct losses or collisions debris typically are the dominating sources for the majority of the LHC areas, significant contribution from beam-gas interactions are expected only in a few areas. Beam-gas interactions are therefore not specifically considered for this analysis but an example of a previous study can be found in [20].

Given the so far short time of operation at low intensities and luminosities, the knowledge of radiation levels for the most critical LHC alcoves is mainly based on simulations. During early operation it is expected that various monitors like for example the RadMon will provide valuable benchmark measurements for the simulation results. This feedback is important as the simulations are associated with significant uncertainties related to for example the beam crossing angle at the interaction point, the actual distribution of beam particle losses around the accelerator, the residual gas density, extrapolation of the cross sections for the primary events, interaction/transport models, and the crucial 


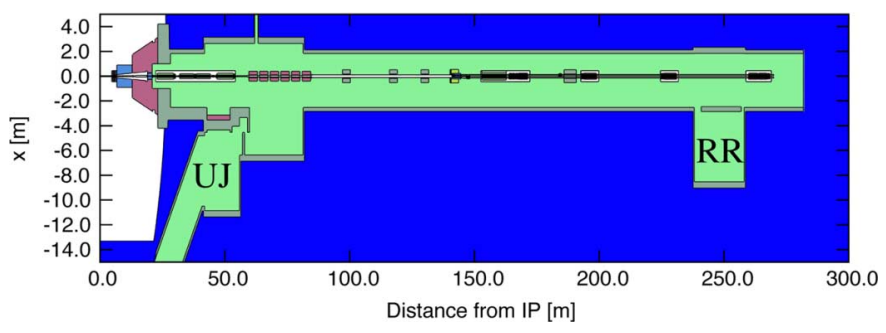

Fig. 4. FLUKA geometry layout for one side interaction point 1.

dependence on a very small angular range of the reaction products. An accurate description of the geometry and material implementation, is also important considering that for example the magnitude of the thermal neutron fluence strongly depends on the actual water content of the concrete walls. At CERF, being a reference facility, the various conditions contributing to the uncertainty are significantly better controlled. For example, samples of the concrete walls have been analyzed in order to implement a more accurate description of the material composition in FLUKA. The thermal neutron fluence has been measured using activation foils and the agreement with FLUKA simulations is within $10-15 \%$.

\section{B. Simulation Results}

The horizontal section at the level of the beam of Interaction Point 1 (IP 1) is shown in Fig. 4. It extends over several hundred meters along the beam line and is implemented with accurate descriptions of all beam line elements like collimators and magnets including magnetic fields. This geometry is a representative example of the geometries used to simulate radiation levels in the LHC. The electronic equipment is installed in dedicated alcoves, typically labelled UJ or RR, and separated from the beam line by various configurations of concrete and/or iron walls. Proton-proton collisions are forced at the interaction point and the emerging particles are transported through the geometry until they either escape the geometry or interact with the beam line leading to electromagnetic and hadronic particle showers. Typically in the order of $10^{5}$ interactions are needed to achieve sufficient statistics in the scoring regions of interest. In addition to the optimization options mentioned in Section II, the simulation is split into smaller units and distributed to a cluster of CPUs. Depending on the CPU specification the calculation time needed per interaction is approximately 10-20 seconds.

In Fig. 5 an example of a typical colormap plot shows how the fluence of high energy hadrons is distributed along the beam line and into the LHC alcoves. The electronic equipment installed in these alcoves may occupy several square meters of floor space. As can been seen in Fig. 5, there are large gradients within these alcoves. It is therefore more appropriate to present the particle fluence as a range to better reflect the local variations and associated uncertainties for the particular installed equipment. The results, normalized to one nominal year of LHC operation, are listed in Table II for some critical LHC areas along with the corresponding 'risk-factor' $\mathrm{R}_{\mathrm{Th}}$. The corresponding neutron lethargy spectra for some LHC alcoves are plotted in Fig. 6. While protons, pions and kaons also will be present, neutrons remain the dominating particle making up $98 \%$ of the high

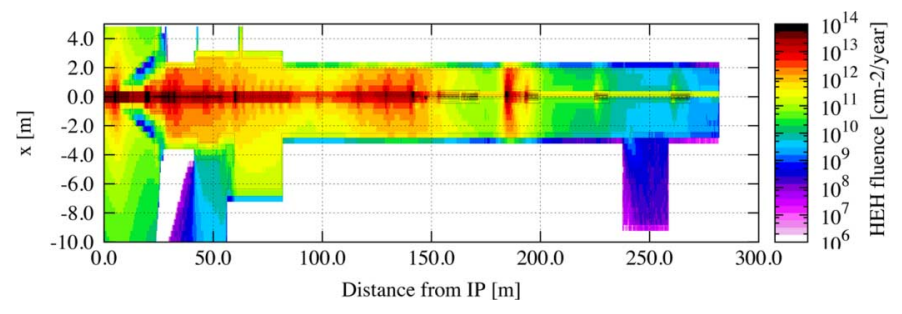

Fig. 5. Scoring map of the high energy hadron fluence (above $20 \mathrm{MeV}$ ) along the beam line for one side of interaction point 1 .

TABLE II

High-ENERgy HADRON FLUENCES AND CORRESPONDING $R_{\text {Th }}$ FOR SOME CRITICAl LHC AREAS. FluenCES ARE NoRMalized to ONE Nominal YEAR OF LHC OPERATION AND GIVEN IN THE UNIT OF PARTICLE FLUENCE PER CM ${ }^{2}$ AND YEAR

\begin{tabular}{l|c|c|c}
\hline \hline $\begin{array}{l}\text { LHC } \\
\text { point }\end{array}$ & Area & $\begin{array}{c}\text { High-energy hadron fluence } \\
{\left[\mathrm{cm}^{-2} \mathrm{y}^{-1}\right]}\end{array}$ & $\mathrm{R}_{T h}$ \\
\hline & Tunnel & up to $1.0 \cdot 10^{10}$ & $3-8$ \\
1 & UJ14/16 & $5 \cdot 10^{8}-5 \cdot 10^{9}$ & 200 \\
5 & UJ56 & $5 \cdot 10^{8}-5 \cdot 10^{9}$ & 1 \\
7 & UJ76 & $2 \cdot 10^{8}-2 \cdot 10^{9}$ & 2 \\
1 & RR13/17 & $1 \cdot 10^{8}-1 \cdot 10^{9}$ & 10 \\
7 & RR73/77 & $2 \cdot 10^{7}-2 \cdot 10^{8}$ & 50 \\
\hline
\end{tabular}

Neutron lethargy spectra for LHC critical areas

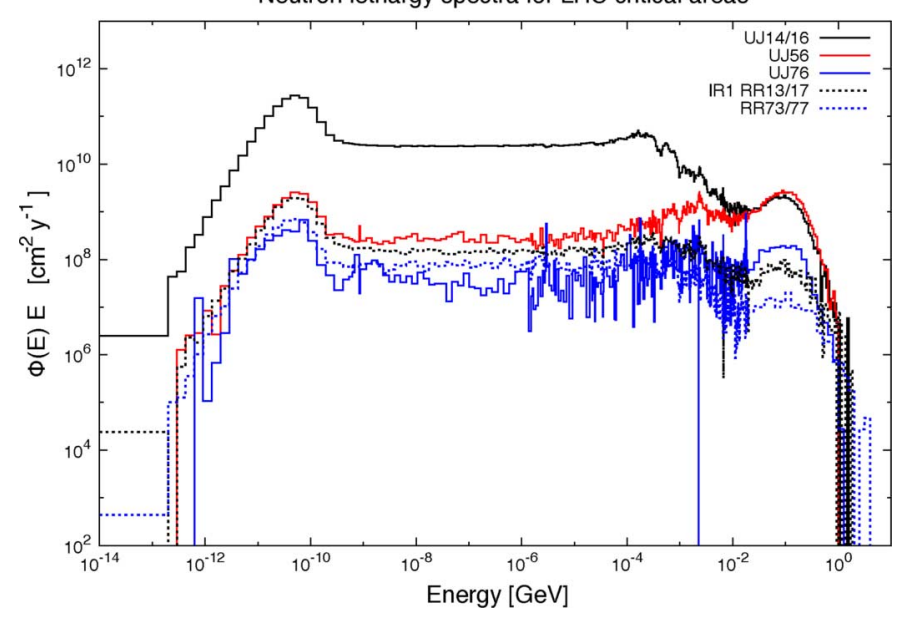

Fig. 6. Neutron lethargy spectra for some critical LHC areas.

energy hadron fluence in the UJ alcoves and 70-90\% in the RR alcoves. As already discussed a potential concern for the equipment installed in the LHC alcoves is their unknown sensitivity to thermal neutrons. In some areas the thermal neutron fluence can reach levels 1-2 orders of magnitude higher than the respective high energy hadron fluence. With thermal neutron cross sections possibly ranging from two to three orders below up to, and even exceeding the high energy hadron cross section [7], it is likely that thermal neutrons can be the dominating factor of SEUs. These simulations show that the risk of thermal neutrons should therefore not be neglected.

As previously discussed in Section III, neutrons from $20 \mathrm{MeV}$ and down to a few MeV may contribute to the SEU rate. In the LHC alcoves the fluence in this energy range is typically a factor 2 lower than the high energy hadron fluence. Though they will not be the dominating factor they should be included for completeness. 


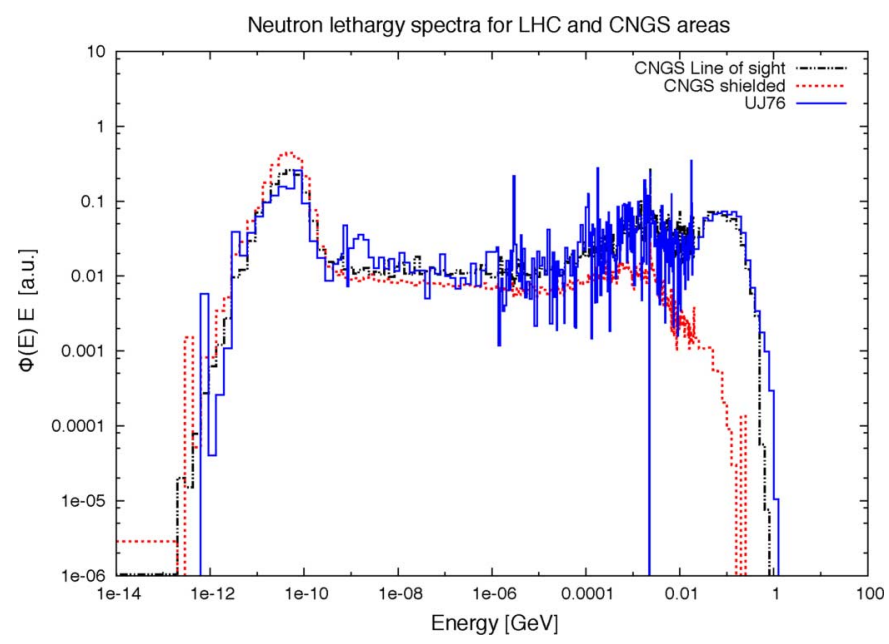

Fig. 7. Neutron spectra for two locations at the CNGS facility and UJ76 alcove in the LHC.

\section{Full System Tests at CNGS}

The FLUKA simulation results do not only provide information about the expected radiation levels in the LHC alcoves, but also allows to better define test criteria for the equipment installed in these alcoves. With the multitude of commercial electronics present in the LHC alcoves, irradiation testing for characterization of single components is practically impossible to achieve. The alternative approach is therefore to carry out full system irradiation tests for indication of failure signatures and rates. However, the availability of appropriate mixed field facilities providing adequate test conditions is very limited. At CERF the layout of the experimental area poses important physical constraints limiting the equipment that can be tested to small systems like the RadMon. Furthermore, only one short test period is available each year limiting the total integrated fluence that can be achieved.

An alternative location at CERN, operating parasitically to the CNGS facility (CERN Neutrinos to Gran Sasso) [21], is for the moment used as a mixed field electronics test area. CNGS uses a high current $450 \mathrm{GeV}$ proton beam hitting a graphite target. Due to the very stable beam conditions, the integrated radiation levels scale with the number of protons on target. In the large side gallery to the graphite target, appropriate test locations have been identified and characterized by FLUKA simulations and measurements. This area is referred to as CNRAD (CNGS Radiation) [22] and as seen in Fig. 7, it can provide energy spectra similar to that of for example the UJ76 alcove. In addition partly shielded test locations can offer high $R_{\text {Th }}$ values, giving the the possibillity to test equipment expected to operate under similar conditions in the LHC.

Recent tests carried out at CNRAD show that some of the commercial equipment installed in the LHC alcoves starts to fail already at high energy hadron fluence of a few $10^{7} \mathrm{~cm}^{-2}$. Considering that high energy hadron fluence in these areas range from $10^{7}-10^{9} \mathrm{~cm}^{-2} \mathrm{y}^{-1}$, and that a large number of equipment is installed, significant failure rates can be expected during nominal operation unless action is taken. However, during the first one to one and a half years of operation the LHC is expected to reach only $1 \%$ of the nominal operating conditions. A one year

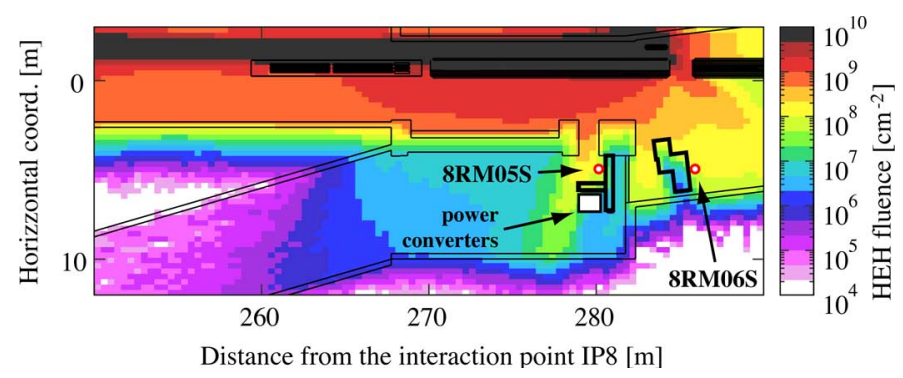

Fig. 8. High energy hadron fluence around the collimator region of TI8. The fluence are normalized for an estimated cumulative annual loss on the respective collimator.

shut-down is then forseen to prepare the machine for nominal operation. Immediate mitigation actions like enforced shielding and relocation of equipment have already been carried out for some of the most concerned areas. An example of how FLUKA can be used to study for example possible improved shielding options can be found in [23]. The gradual increase in intensity and luminosity, together with the immediate and additional mitigation actions planned for the first shut-down period, will therefore contribute to reduce the probability of failures due to SEUs [19].

\section{LHC APPliCATION CASE StUdy AND FIRST BENCHMARK}

Before being injected into the LHC, the proton beam is accelerated to $450 \mathrm{GeV}$ in the SPS accelerator (Super Proton Synchrotron). Extraction of the SPS beam is carried out in two different locations in order to have two counter rotating beams in the LHC. At the injection points (TI2, TI8) significant radiation levels are expected due the beam interacting with injection line collimator. In the nearby UJ87 alcove a number of power converters are installed close to the open access linking the UJ87 to the beam line and tunnel area. Monte Carlo simulations have shown that this location can be exposed to a high energy hadron fluence in the order of $10^{7} \mathrm{~cm}^{-2}$ for an estimated cumulative annual loss on the collimator. A distribution of the high energy hadron fluence is shown in Fig. 8 marking also the location of the power converters. In a recent test at CNRAD where only one part of these power converters where tested, a destructive failure was experienced after a high energy hadron fluence of a few $10^{8} \mathrm{~cm}^{-2}$. Even though the $40 \mathrm{~cm}$ thick iron wall placed just in front of the power converters will partly reduce the radiation streaming through from the tunnel, it is not sufficient to keep a safety margin of at least a factor 10 . It has therefore been suggested that the shielding of UJ87 should be enforced closing the access opening with a movable plug. This is a mitigation option that requires both a significant investment in shielding blocks in addition to a large intervention from the LHC integration team. Providing a comparison to measurements is therefore important to support the simulation results. For this purpose two RadMon locations (8RM05S, 8RM06S, see Fig. 8), one inside and one outside of the UJ87 have been used. The number of SEUs measured by each RadMons was registered during the setup procedure of the collimator with a known number of protons impinging on its jaw. Applying the approach explained in Section III, these measurements agree well with Monte Carlo based predictions as can be seen in Table III. For the inside position 
TABLE III

Number of SEUs MEASURED FOR Two RADMON LOCATIONS, DURING THE SETUP OF THE TI8 INJECTION LINE COLLIMATOR, COMPARED TO FLUKA PREDICTIONS. THE ERROR OF THE PREDICTION INCLUDES THE STATISTICAL UNCERTAINTIES OF THE SIMULATION RESULTS IN ADDITION TO UNCERTAINTIES Associated With the SEU Bit Cross Section VAlues. Counting STATISTICS IS APPLIED FOR THE RADMON MEASUREMENT RESULTS

\begin{tabular}{ccc}
\hline \hline Location & RadMon measurement & FLUKA prediction \\
\hline 8RM05S (3V) & $12 \pm 3.5$ & $11.5 \pm 2.3$ \\
8RM06S $(5 \mathrm{~V})$ & $1 \pm 1$ & $0.9 \pm 0.2$ \\
\hline \hline
\end{tabular}

thermal neutrons are expected to be the dominating contributor as this device were operated at $3 \mathrm{~V}$ and the simulated $\mathrm{R}_{\mathrm{Th}}$ is approximately 50 . As the thermal neutron fluence is more evenly distributed compared to the higher gradient of the high energy hadron fluence, this makes the measurement less sensitive to how accurately the RadMon is positioned.

For the outside position where the RadMon operates at $5 \mathrm{~V}$ the ratio of thermal neutron fluences to high energy hadron fluence is even higher at a value of 150 . Thus, even though the sensitivity to thermal neutrons at $5 \mathrm{~V}$ is a factor ten lower than at $3 \mathrm{~V}$, thermal neutrons will probably still be dominating. This measurement is limited by the low number of protons lost on the collimator resulting in only 1 count for the available test period. It is therefore likely that if the measurement were to be repeated for the same number of lost protons, the result could be 2, 3 or even no counts at all. However, a measurement of 1 count is not an order of magnitude off the prediction, and the inside location also shows very good agreement given the exact same beam conditions. This indicates a preliminary confidence in the comparison. As the LHC continues operation more data will eventually be collected to provide a better benchmark, not only in this area but in all other areas where RadMons are installed.

\section{SUMMARY}

This paper has presented FLUKA simulation results of particle energy spectra and fluences for critical LHC areas. For nominal operation of the LHC, equipment installed in the LHC alcoves is expected to be exposed to a mixed radiation field with high energy hadron fluences ranging from $10^{7}$ up to some $10^{9}$ $\mathrm{cm}^{-2} \mathrm{y}^{-1}$. Combined with full system test at the CNRAD test area, FLUKA simulations have helped to identify critical areas where mitigation options have to be implemented in order to reduce failure probabilities. The simulations have further shown how some areas have a high ratio of thermal neutrons to high energy hadrons, potentially risking that thermal neutrons may be the dominating source of failure.

The approach of comparing RadMon measurements to FLUKA predictions has been benchmarked in a mixed field environment at CERF and further shown good preliminary results for the LHC environment. A dedicated monitoring and calculation working group has recently been formed at CERN in order to systematically collect more data from the several hundreds of RadMons installed at LHC. As LHC continues its operation towards nominal luminosity this will provide improved benchmark studies not only for SEUs but also for total ionizing dose and displacement damage.

\section{ACKNOWLEDGMENT}

The authors would like to thank the FLUKA team for contributions to simulations of the LHC radiation levels, the CERN Radiation Protection group for providing the FLUKA geometry model of the CERF facility, the CNGS collaboration for providing the FLUKA geometry model of the CNGS facility, and the CERN RadMon team for providing RadMon devices for the measurements.

\section{REFERENCES}

[1] M. Huhtinen and F. Faccio, "Computational method to estimate single event upset rates in an accelerator environment," Nucl. Instrum. Methods Phys. Res. A, vol. A450, no. 1, pp. 155-172, 2000.

[2] I. Dawson and C. Buttar, "The radiation environment in the atlas inner detector," Nucl. Instrum. Methods Phys. Res. A, vol. A453, no. 1-2, pp. 461-467, 2000

[3] I. Dawson, C. Buttar, N. Mokhov, A. Moraes, and M. Shupe, "Predicting the radiation environment at high-luminosity hadron-collider experiments," Nucl. Instrum. Methods Phys. Res. A, vol. A510, no. 3, pp. 219-232, 2003.

[4] B. Pastirčák, "FLUKA Monte Carlo simulation code as used for radiation studies in the alice experiment," Phys. Particles Nuclei Lett., vol. 5, pp. 301-304, 2008.

[5] A. Morsch and B. Pastirčák, "Radiation in ALICE detectors and electronic racks," ALICE Internal Note, ALICE-INT-2002-028, 2002.

[6] O. S. Brüning, P. Collier, P. Lebrun, S. Myers, R. Ostojic, J. Poole, and P. Proudlock, LHC Design Rep., CERN, Geneva, Switzerland, 2004.

[7] E. Normand, K. Vranish, A. Sheets, M. Stitt, and R. Kim, "Quantifying the double-sided neutron seu threat, from low energy (thermal) and high energy (gt; $10 \mathrm{mev)} \mathrm{neutrons,"} \mathrm{IEEE} \mathrm{Trans.} \mathrm{Nucl.} \mathrm{Sci.,} \mathrm{vol.} \mathrm{53,}$ no. 6, pp. 3587-3595, Dec. 2006.

[8] A. Ferrari, J. Ranft, and P. R.' Sala, "The FLUKA radiation transport code and its use for space problems," in Proc. 1st Int. Workshop Space Radiation Research and 11th Annu. NASA Space Radiation Health Investigators' Workshop, Arona, Italy, May 2000.

[9] A. Ferrari, P. Sala, A. Fassó, and J. Ranft, "FLUKA: A multi-particle transport code," CERN-2005-10-2005, INFN/TC_05/11, SLAC-R-773.

[10] G. Battistoni, S. Muraro, P. R. Sala, F. Cerutti, A. Ferrari, S. Roesler, A. Fassó, and J. Ranft, "The FLUKA code: Description and benchmarking," in Proc. Hadronic Shower Simulation Workshop, M. Albrow and R. Raja, Eds., Sep. 6-8, 2006, Fermilab, AIP Conf. Proc. 896, 31-49 (2007)

[11] F. Ballarini, G. Battistoni, M. Brugger, M. Campanella, M. Carboni, F. Cerutti, A. Empl, A. Fassó, A. Ferrari, E. Gadioli, M. V. Garzelli, M. Lantz, A. Mairani, A. Mostacci, S. Muraro, A. Ottolenghi, V. Patera, M. Pelliccioni, L. Pinsky, J. Ranft, S. Roesler, P. R. Sala, D. Scannicchio, G. Smirnov, F. Sommerer, S. Trovati, R. Villari, V. Vlachoudis, T. Wilson, and N. Zapp, "The physics of the fluka code: Recent developments," Adv. Space Res., vol. 40, no. 9, pp. 1339-1349, 2007.

[12] M. Brugger, F. Cerutti, L. Lari, M. Mauri, S. Roesler, L. Sarchiapone and V. Vlachoudis, "LHC accelerator design studies on the example of passive absorbers," Nucl. Technol., vol. 168, no. 3, pp. 659-664, Dec. 2009.

[13] R. C. Baumann, "Radiation-induced soft errors in advanced semiconductor technologies," IEEE Trans. Device Mater. Reliab., vol. 5, no. 3, pp. 305-316, Sep. 2005.

[14] H. H. K. Tang, "Nuclear physics of cosmic ray interaction with semiconductor materials: Particle-induced soft errors from a physicists perspective," IBM J. Res. Develop., vol. 40, no. 1, pp. 2162-2167, 1996.

[15] A. Mitaroff and M. Silari, "The CERN-EU high-energy reference field (CERF) facility for dosimetry at commercial flight altitudes and in space," Radiat. Protect. Dosim., vol. 102, pp. 7-22, 2002.

[16] T. Wijnands, C. Pignard, and R. Tesarek, "An on line Radiation monitoring system for the LHC machine and experimental caverns," in Proc. 12th Workshop Electronics for LHC and Future Experiments, 2006.

[17] R. Harboe-Sorensen, C. Poivey, F.-X. Guerre, A. Roseng, F. Lochon, G. Berger, W. Hajdas, A. Virtanen, H. Kettunen, and S. Duzellier, "From the reference seu monitor to the technology demonstration module on-board proba-ii," IEEE Trans. Nucl. Sci., vol. 55, no. 6, pp. 3082-3087, Dec. 2008. 
[18] D. Kramer, M. Brugger, V. Klupak, C. Pignard, K. Røed, G. Spiezia, L. Viererbl, and T. Wijnands, "LHC RadMon SRAM detectors used at different voltages to determine the thermal neutron to high energy hadron fluence ratio," Proc. RADECS, 2010.

[19] M. Brugger, "Review of critical radiation areas for LHC electronics and mitigation actions. radiation monitoring and first results," in Proc. CERN LHC Performance Workshop, Chamonix, France, 2010.

[20] A. Butterworth, A. Ferrari, E. Tsoulou, V. Vlachoudis, and T. Wijnands, "Estimate of radiation damage to low-level electronics of the RF system in the LHC cavities arising from beam gas collisions," $R a$ diat. Protect. Dosim., vol. 116, no. 1-4, pp. 521-524, Dec. 20, 2005.
[21] CERN Neutrinos to Gran Sasso [Online]. Available: http://proj-cngs. web.cern.ch/proj-cngs

[22] CNRAD [Online]. Available: http://cngs-rad-facility.web.cern.ch/ cngs-rad-facility

[23] R. Versaci, K. Røed, M. Brugger, A. Mereghetti, F. Cerutti, V. Vlachoudis, and F. Ferrari, "R2e fluka studies for point 1 and point 5," CERN-ATS-Note-2010-046 PERF, 2010. 